\title{
UNSUPERVISED TEXTURE IMAGE SEGMENTTATION BY IMPROVED NEURAL NETWORK ART2
}

\author{
Zhiling Wang, G. Sylos Labini, R. Mugnuolo and Marco De Sario ${ }^{\dagger}$ \\ Center for Space Geodesy, Italian Space Agency, P.O. Box II \\ 75100 Matera, Italy \\ Fax:+39-835-339005 Tel:+39-835-3779 Email:zhiling@asimto.mtasi.it \\ †Dept. of Electronic Engineering. University of Bari, Via Re Datid,200 \\ 70125 Bari, Italy
}

\begin{abstract}
We here propose a segmentation algorithm of texture image for computer vision system on space robot. An improved Adaptive Resonance Theory (ART2) for analog input patterns is adapted to classify the image based on a set of texture image features extracted by a fast Spatial Gray Level Dependence Method (SGLDM). The nonlinear thresholding functions in input layer of the neural network have been constructed by two parts: firstly to reduce the effection of image noises on the features, a set of sigmoid functions is chosen depending on the types of the feature; secondly, to enhence the contrast of the features, we adopt fuzzy mapping functions The cluster number in output layer can be increased by an autogrowing mechanism constantly when a new pattern happens. Experimental results and orginal or segmented pictures are shown, including the comparison between this approach and $\mathbf{K}$-means algorithm. The system written by C language is performed on a SUN-4/330 sparc-station with an image board IT- I50 and a CCD camera.
\end{abstract}

\section{Introduction}

Segmentation and classification of textured images have been considerable attention to contain significant discriminatory information for image segmentalion in a variety of application, such as terrain classific ation, military surveillance and recognition, remote sensing inages and biomedical image analysis ${ }^{2}$. Although texture is a fundamental characteristic of inages, the complexity involved in its quantification has presented its effective incorporation into the segmentation process.

in this paper, the neural network of an improved Adaptive Resonance Theory (ART2) is presented to segment an inage consisting of several regions with different textures. Artificial neural networks offer several advantages over conventional classification techniques, due to their high computation rate, great degree of fault tolerance and unsupervised ability. The number of researches have engaged on the researchment by neural networks ${ }^{20 \sim 31}$

In this paper, section 2 defines the texture feature types which are derived from co-occurrence matrixes and selection of maximum and minimum measure window for feature extraction of the lexture image. Section 3 describes an approach of improved ART2 neural network with alterable competitive layer $\left(F_{2}\right.$ laver $)$. The nonlinear thresholding fuction in $F_{1}$ layer is displaced by a fuzzy mapping function. Section 4 shows the results of experiments and illustration.

\section{Feature extraction of texture image}

Whether the segmentation of texture image is good or not depends on the extraction of texture features. There are number of the approaches to have been developed for feature extraction of the texture image: Fourier power spectrum method (FPSM) ${ }^{3}$,spatial texiure energy ${ }^{5}$, Markov randon field model ${ }^{7}$, Gibbs random field mode $l^{8,9}$, zero-sum filter masks ${ }^{14}$, gray level run length method (GLRLM) 10, spatial gray level dependece method (SGLDM) ${ }^{2}$, gray level difference method (CLDM) ${ }^{11}$, and other methods $6,12,13,15$. Sonte of these methods belong to statistical method, others to structural one. Among them, spatial gray level dependence method, which is introduced by Haralick et al. in their paper ${ }^{2}$, is one of the most successful statistical representation for the texture. The feature measurement from co-occurrence matrices in the SCLDN is rather similar to the knowledge captured by the human eyes, and provides a convenient way to represent the properties of object textures. Weszka et at. experinentally compared feature on terrain innges and found that SGLDM is more powerful than the GLDM, GRLM, and FPSM ${ }^{1}$; Ohanian $t \mathrm{al}$. also point ted that the features by SGLDM were better than Markov random field, multi-channel filtering features, and fractal based features ${ }^{16}$. It is known, however, that the SGLDM requires much processing time and great number of memory. Only for mean probability distribution, $2^{34}$ times of nultiplication in the SCLDM are done when a measured inage is a size $64 \times 04$ with gray level 128 , and the tenclency will be raised at exponent rate with the enlargemput of the image size, particularly, the increase of gray-level number.

In this paper, we use a set of simplifed equations based on a fact that rows or columns around the current pixel are included or excluded almostly at the same time while the measured window is displaced in the horizontal or vertical direction of the image, so we could make the equation be simplifed viewing from the pixels of rowa(columns) both excluded and included from a window rather than a pixel method ${ }^{4}$. Some calculations are done one time in a row or column instead of one in a pixel, so algorithm in the paper consumes much less time than Harilick's method.

We defiled a co-occurrence matrix of relative frequencies with which two pixpls separated by distance $d$ at a specified angle orcur on the image, one witl hray level $i$ and the of her with gray level $j$

199. by the Anerican Institute of Aeronautics and Astronautics, Inc. All rights reserved. 
A distance of one pixel, i.e. the measuring window slides over the image in one step length ${ }^{1}$ in both horizontal and vertical direction and angle quantixed to $45^{\circ}$ intervals, or $0^{\circ}, 45^{\circ}, 90^{\circ}$, and $135^{\circ}$ will be used. We give a set of simplified equations:

(1) Mean

$$
m_{k}=m_{k-1}+\frac{1}{N} \sum\left(i_{k, r}^{+}+j_{k, r}^{+}-i_{k, r}^{-}-j_{k, r}^{-}\right)
$$

(2) Variance

$$
\begin{aligned}
\sigma_{k}^{2}= & \sigma_{k-1}^{2}+ \\
& \frac{1}{N} \sum\left[\left(i_{k, r}^{+}\right)^{2}+\left(j_{k, r}^{+}\right)^{2}-\left(i_{k, r}^{-}\right)^{2}-\left(j_{k, r}^{-}\right)^{2}\right]+m_{k-1}^{2}-m_{k}^{2}
\end{aligned}
$$

(3) Correlation

$$
\begin{aligned}
C_{k}= & {\left[C_{k-1} \sigma_{k-1}^{2}+\right.} \\
& \left.\frac{2}{N} \sum\left(i_{k, r}^{+} j_{k, r}^{+}-i_{k, r}^{-} j_{k, r}^{-}\right)+m_{k-1}^{2}-m_{k}^{2}\right] / \sigma_{k}^{2}
\end{aligned}
$$

(4) Energy

Let

$$
\begin{gathered}
L^{-}=\frac{N_{k-1}}{N_{k-1}-2}, \quad J^{-}=1 /\left(N_{k-1}-2\right) \\
L^{+}=\frac{N_{k-1}}{N_{k-1}+2}, \quad J^{+}=1 /\left(N_{k-1}+2\right) \\
E_{k}^{-}=\left(L^{-}\right)^{2} E_{k-1}^{-}+\left(J^{-}\right)^{2}\left(a-4 M_{k-1}^{-}\right) \\
E_{k}^{+}=\left(L^{+}\right)^{2} E_{k-1}^{+}+\left(J^{+}\right)^{2}\left(a+4 M_{k-1}^{-}\right)
\end{gathered}
$$

where

$$
a= \begin{cases}2 & \text { if } i \neq j \\ 4 & \text { if } i=j\end{cases}
$$

(5) Entropy

$$
\begin{gathered}
E P_{k}^{-}=L^{-} E P_{k-1}^{-}-L^{-} \log \left(L^{-}\right)-A^{-} \\
E P_{k}^{+}=L^{+} E P_{k-1}^{+}-L^{+} \log \left(L^{+}\right)-A^{+} \\
A^{-}=\left\{\begin{array}{l}
2 J^{-}\left(M_{k-1}\left(i_{k, r}^{-}, j_{k, r}^{-}\right)-1\right) \log \left(L^{-}\left(M_{k-1}\left(i_{k, r}^{-} j_{k, r}^{-}\right)-1\right)+\right. \\
2 J^{-} M_{k-1}\left(i_{k, r}^{-}, j_{k, r}^{-}\right) \log \left(L^{-} M_{k-1}\left(i_{k, r^{-}}^{-} j_{k, r}^{-}\right)\right) \\
J-\left(M_{k-1}\left(i_{k, r}^{-} j_{k, r}^{-}\right)-2\right) \log \left(L^{-}\left(M_{k-1}\left(i_{k, r}^{-} j_{k, r}^{-}\right)-2\right)+\right. \\
J-M_{k-1}\left(i_{k, r}^{-}, j_{k, r}^{-}\right) \log \left(L-M_{k-1}\left(i_{k, r}^{-} j_{k, r}^{-}\right)\right. \\
A^{+}=\left\{\begin{array}{l}
2 J^{+}\left(M_{k-1}\left(i_{k, r}^{+}, j_{k, r}^{+}\right)+1\right) \log \left(L^{+}\left(M_{k-1}\left(i_{k, r}^{+}, j_{k, r}^{+}\right)+1\right)\right. \\
2 J^{+} M_{k-1}\left(i_{k, r}^{+}, j_{k, r}^{+}\right) \log \left(L^{+} M_{k-1}\left(i_{k, r}^{+}, j_{k, r}^{+}\right)\right. \\
J^{+}\left(M_{k-1}\left(i_{k, r}^{+}, j_{k, r}^{+}\right)+2\right) \log \left(L^{+}\left(M_{k-1}^{+}\left(i_{k, r}^{+}, j_{k, r}^{+}\right)+2\right)\right. \\
J^{+} M_{k-1}\left(i_{k, r}^{+}, j_{k, r}^{+}\right) \log \left(L^{+} M_{k-1}\left(i_{k, r}^{+}, j_{k, r}^{+}\right)\right.
\end{array} \quad \mathrm{i} \neq j\right.
\end{array}\right.
\end{gathered}
$$

Ordinarily, rather than using a single displacement berause small values for step length d yield the best results for the extraction of image features proved
bv Weszla at. at.
(6) Contrast

$$
\left.T_{k}=T_{k-1}+\frac{2}{N} \sum\left[\left(i_{k, r}^{+}-j_{k, r}^{+}\right)^{2}-\left(i_{k, r}^{-}-j_{k, r}^{-}\right)^{2}\right)\right]
$$

(7) Homogeneity

$$
\begin{aligned}
H_{k}= & H_{k-1}+ \\
& \frac{2}{N} \sum\left\{\left(1+\left(i_{k, r}^{+}-j_{k, r}^{+}\right)^{2}\right)^{-1}-\left(1+\left(i_{k, r}^{-}-j_{k, r}^{-}\right)^{2}\right)^{-1}\right)
\end{aligned}
$$

where the $\sum$ is the $\sum_{r=1}^{L}$, the $L$ stands for the length of the row or column, i.e the wide of measuring square window, the $M(i, j)$ is the element of a co-occurrence matrix, superscipts " $t$ " and "-" express for a pixel $(x, y)$ included or excluded from the window. The equations for both energy and entropy features are used to the case considering a pixel included or excluded from the window because of the nonlinear decomposition for square and logarithm functions

\section{Improved ART2}

Comectionist classification used here is called Adaptive Resonance Theory(ART) ${ }^{24 \sim 27}$. In general, $A \overrightarrow{R T}$ is divided into two types depending on input patterns. ART1 is applied to solve binary input problem, ART2 is available to both binary and analog inputs. In the paper, the ART2 is used to classify the texture image because the 20 features (five for each angle) belong to gray-scale patterns.

The classifier in the ART2 consists nuainly of two subsystems: the attentional subsystem and the orienting subsystem. The former is composed of the Short-Term Memory (STM) and Long-Term Memory (LTM) elements.

\subsection{Short-Term Memory (STM)}

The $F_{1}$, the input representation field, and $F_{2}$, the category representation field(competitive nechanism), are the two STM nain components.

$F_{1}$ is composed of three layers with STM activation equations as (see Fig. 1)

$$
\begin{gathered}
p_{i}=u_{i}+\sum g\left(y_{j}\right) Z_{j} \\
q_{i}=\frac{p_{i}}{e+\|p\|} \\
v_{1}=f\left(x_{i}\right)+b f\left(q_{1}\right) \\
u_{i}=\frac{v_{i}}{e+\|v\|} \\
w_{i}=I_{i}+a u_{i} \\
x_{i}=\frac{w_{i}}{e+\|w\|}
\end{gathered}
$$

where $a, b$, and e are constants, $y_{1}$ is the STM activation of the Jth $F_{2}$ newron, \|\| is the $L_{2}$ norm, $f()$ is a nonlinear threshold function:

$$
f(x)= \begin{cases}0 & \text { for } \theta \leq x \leq \theta \\ 2\left(\frac{r-\beta}{\beta-\theta}\right)^{2} & \text { for } \theta \leq x \leq \alpha \\ 1-2\left(\frac{x-\beta}{\beta-\theta}\right)^{2} & \text { for } \alpha \leq x \leq \beta \\ 1 & \text { for } x \geq \beta\end{cases}
$$

where the feature noises are suppressed by seting $f(x)$ to zero when $0 \leq x<\theta$. The fuzzy mapping function is used to enhence the contrast amone the features. and makes the invut vatterns classified 


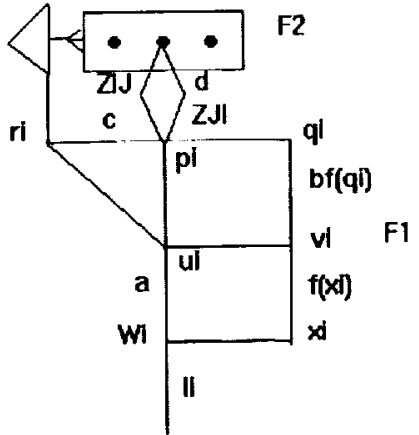

Fig. 1. Typical ART2 arclitecture $25,26,27$

more easily. The normalization mechanism keeps the pattern from saturation in spite of the constant presence of the pattern during the learning process. The $F_{1}$ layer provides internal feedback and a correlation between normalized bottom-up and top-down patterns to stabilize all activities in the STM before transmitting the output of the $F_{1}$ layer to the $F_{2}$ layer.

\subsection{The search phase}

In the $F_{2}$, a competitive mechanism is used to choose a winning neturon. Firstly, the input pattern of the $F_{1}$ is applied to the bottonup adaptive filter by the bottom-up adaptive weight $Z_{i j}$.

$$
T,=\sum p_{1} Z_{1 j} \quad \text { for } j=1,2, \ldots, K
$$

where $\mathrm{K}$ is the total number of existing categories in the $F_{2}$, then the vector $\mathbf{T}$ is put in the order fron minimum value to maxinum one. We here suppose the Jth neuron in the $F_{2}$ is selected if this neuron becones maximally active one anong the neurons not to be reset in the trial, i.e.

$$
T_{J}=\max \left(T_{j}\right) \quad j=1,2, \ldots, K_{J}
$$

where $K_{J}$ is the total number of the categories not to be used, then only winning neuron in the $F_{2}$ has nonzero outputs.

$$
g\left(T_{j}\right)= \begin{cases}d & \text { if the Jth } F_{2} \text { neuron is the winner based on } \\ & \text { max }\left(\sum p_{i} Z_{i j}\right) \text { and it has not been reset in the trial } \\ 0 & \text { otherwise }\end{cases}
$$

The top-down pattern $g\left(T_{j}\right)$ is then feedback to the $F_{1}$ by top-down adaptive weight $Z_{j i}$ and compared to the original bottom-up pattern to see if a correct match has been made by an activated orienting subsysten.

\subsection{Orienting subsystem}

The orienting subsystem helps to directly search for the categories in the $F_{2}$. When the subsvstem is activated, the bottom-up pattern vector $\mathbf{p}$ and the top-down pattern vector $\mathbf{u}$ are utilized to calculate the degree of natich (vector $r$ )

$$
r_{k}=\frac{u_{i}+c p_{1}}{e+\|u\|+\|c p\|}
$$

if the choise in the $F_{2}$ is correct, i.e.

$$
\text { (I) } r \|>\rho
$$

where $\rho$ stands for the vigilance factor or match sensitivity parameter. At this time, adaptive resonance is considered to have occured and entered to the categories in the Long-Term Menory (LTM). If the choice is incorrect, another neuron with maximum output value among the existing neurons not to be selected will be selected as a possible winner candidate. The new caudidate may cause yet another mismatclı, lience another reset happens and the selection of yet another neuron, eventually, either the bottom-up pattern will be placed in an existing category or learned as the first example of a new category in the $F_{2}$ layer. It is possible for an autogrowing mechanism to be activated to create a new catogory if no category in the $F_{2}$ could be used to save the new one.

\subsection{The Long-Term Memory (LTM)}

The LTM is made up of two components, the bottom-up adaptive weight $Z_{i j}$ and the top-down adaptive weight $Z_{j i}$. When the match operation in the orienting subsystem occures successfully, the botton-up and top-down weights should be adjusted. The weights can been obtained easily by

$$
\begin{aligned}
& Z_{t J}=\frac{u_{i}}{I-d} \\
& Z_{J t}=\frac{u_{i}}{I-d}
\end{aligned}
$$

The procedure in the improved ART2 can be summarized as: Step 1. Initialize bottom-up and top-down adaptive weights $Z_{i j}$ and $Z_{j i}$ in the LTM.

Step 2. Apply a new input pattern.

Step 3. Stabilize the output vectors $u$ (or $p$ ) of the $F_{1}$ layer by repeated operating Eqs. $11 \sim 16$, including noise reduction and contrast enhencement by a nonlinear thresholding function and fuzzy mapping function.

Step 4. Compute the output vector $p$ by Eq. 18.

Step 5. Select a winner neuron by Eqs. 18 and 19 if neurons not to be selected exist, else go to step 7 .

Step 6. Apply Eq. 21 to determine whether the selected top-down winner pattern matches the bottom-up input $\mathbf{u}$ within a certain acceptance level of vigilance. if $\mathbf{E q} .21$ is not true, the selected winner neuron in the $F_{2}$ is disabled and return to step 5 in order to choose another winner neuron; else go to step 8;

Step 7. Autogrowing mechanism is activated to create a new category.

Step 8. Only adjust the bottom-up and top-down adaptive weights with respect to the matched winner neuron by Eqs. 23 and 24.

Step 9. Before taking the next new input pattern, neuron which has been disable int step 6 will be enabled. The process return to step 2 if a new input pattern at least exists, else exit the system.

Viewing from the improved ART2 algorithm, if the network for an input patten has learned previously to recognize the pattern, then 


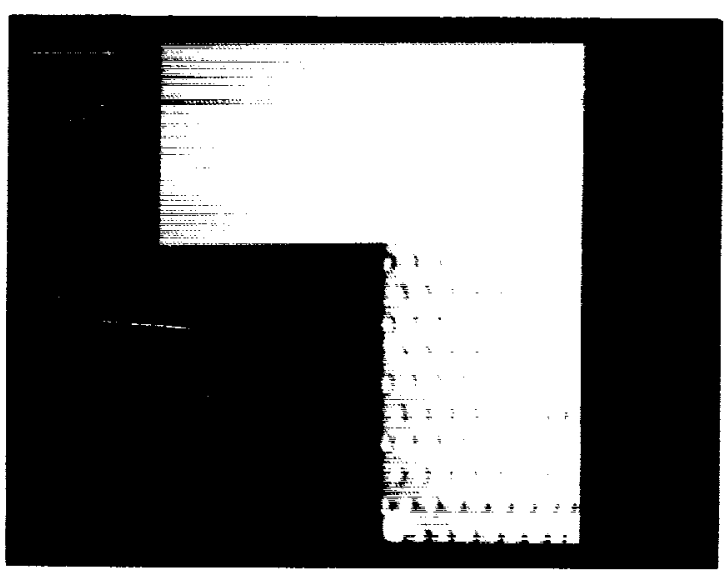

(a)

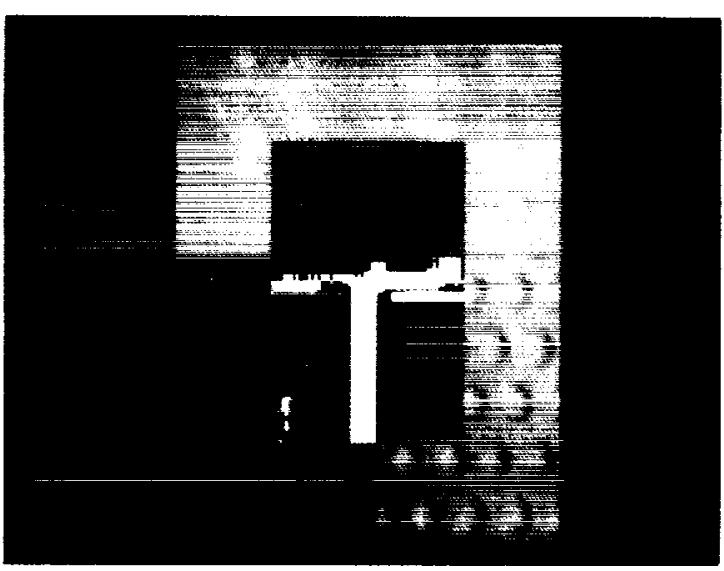

(c)

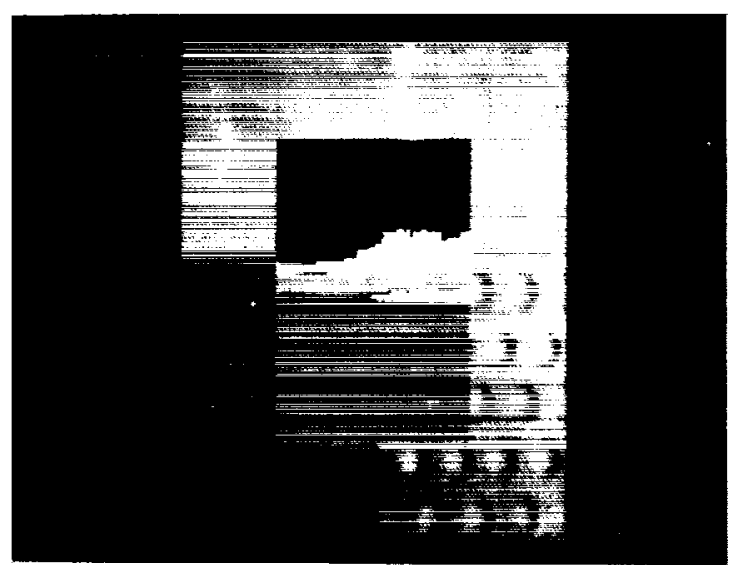

(b)

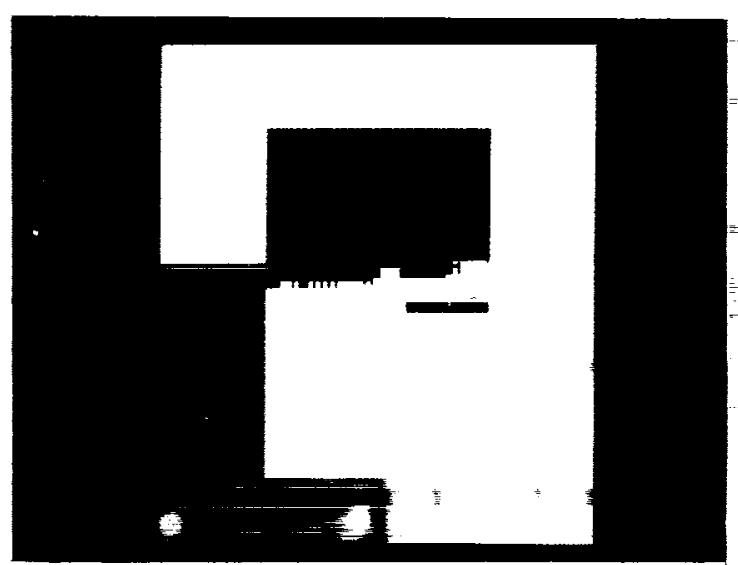

(d)

Fig. 2. (a) original, natural texture image, (b) segmentation by K-means algorihm, (c) energy segmentation by the improved ART2 only with noise reduction, (d) energy segmentation by the improved ART2 with both noise reduction and contrast enhencement.

a resonant state will be achieved quickly when that pattern is presented, adaptive process will reinforce the memory of the stored pattern by formulas. If the pattern is not immediately recognized, the network witl rapidly search through its stored patterns looking for a match. If no match is found, it will enter a resonant state whereupon the pattern will be stored as a new category for the first time if unused neurons in the competitive layer exist. Otherwise, a new neuron shoukl be created automatic ally by the aulogrowing mechanism the $F_{2}$ layer to store the new pattern. Thus, the network is able to respond fastly 10 previonsly learned data, yet learn novel data when those are presented. 


\section{Experiment results}

The performance of the segment algorithm by improved ART2 is examined by a series of experiments on image containing different textures. The size of each image is $100 \times 100$ with 256 gray levels. The size of maximum and minimum measuring window is defined as $11 \times 11$ and $33 \times 33$, respectively.

For the texture features from the image by fast SGLDM algorithm, the K-means algorithm is used ${ }^{17}$ (shown in Fig. 2 (b)) However, the $\mathbf{K}$-means algorithm has following disavantages:

- Supervised learning mode: the number of clusters must be set in advance. the different number may classify different results

- Slow real-time ability: time of classification will raise at exponent rate with the cluster number increased;

- Unstability: the results of classification depends on the precision of feature extraction. when the extraction of the texture features has slightly change, the classifing result might be difference.

Compared to the K-means algorithm, the ART2 has many advantages, such as unsupervised training, high computation rates, and great degree of fault tolerance (stalility/plasticity).

In our test, the features, i.e. energy, entropy, correlation, homogeneity and inertia (or called as contrast), are used in texture analysis. The features have been proved to be a high degree of accuracy for the extraction of texture image features ${ }^{3}$. The parameters $a, b, c, d, e, \theta$ and $\rho$ is selected in advance. $a=b=10, c=0.25, d=0.8$ $e=10^{-6}$. the selection of $\theta$ depends on different texture features and quantized angles of the features. For instance, the noise of each angle for the energy feature in the test is sinuilar, so the value of $\theta$ is selected as 0.23 in every angle of the feature. On the other hand, the noise of each angle for the contrast feature is slightly different. the $\theta$ is set to $0.1,0.12,0.2$, and 0.1 for the feature along to angle $0^{\circ}, 45^{\circ}$, $90^{\circ}$, and $135^{\circ}$, respectively. The Fig 2 . (a) is the original texture image. The Fig. 2. (c) is the segmenting result of the improved ART2 only with noise reduction. It is seen from the Fig. 2. (c) to greatly improve the segmentation of the texture inage. The Fig. 2. (d) shows that the segmentation operation is further good after not only the noise reduction but also the feature enhencement are done.

\section{Conclusion}

The SGLDM provides the most powerful statistical represeutation for segmentation and identification of texture images. Its problem, consuming time has been improved greatly by a fast algorithm.

An improved Adaptive Resonance Theory (ART2) for analog input patterns is adapted to classify the image based on a set of texture innage features extracted by a fast SGLDM. The non-linear thresholding functions in the ART2 $F_{1}$ layer bave been composed of two parts: to reduce the effection of image noises on the features, a set of sigmoid functions is chosen depending on the types of the feature; to enhence the contrast of the features, we adopt fuzzy multi-region mapping functions The cluster number in output layer can be increased by an autogrowing mechanism constantly when a new pattern happens. The system written by $\mathrm{C}$ language is performed on a SUN-4/330 sparc-station with an image board IT-150 and a CCD camera.

\section{Acknowledgements}

The authors acknowledge the discussion made by A. Acciani and G. Bianco to provide us wiht $\mathrm{C}$ library.

\section{References}

[1] Van Gool L., P. Dewaele, and A. Oosterlinck (1985). Texture analysis anno 1983. Comput. Vision Craphics Image Processing $29,336-357$

[2] Haralick R. M., K. Shanmugan, and I. Dinstein (1973). Texture features for image classification. IEEE Trans. on Syst., Man, and Cybernet. 3. 610-621.

[3] Weszka J. S., C. R. Dyer, and A. Rosenfeld (1976). A comparative study of texture measures for terrain classification. IEEE Trans. on Syst, Mam, and Cybernet. 6. 269-285.

[4] Lee J. H. and N. H. Lee (1992). A fast and adaptive method to estimate texture statistics by the spatial gray level dependence matrix (SCLDM) for texture image segmention. Peftern Recog. Letters 13. 291-303.

[5] Laws K. L. (1980). Rapid texture identification. Proc. SPIE 238. 376-380.

[6] Therrien C. W. (1983). An estimation-theoretic approach to terrian image seginentation. Comput. Vision Graphics Image Pro. cessing $22.313-326$.

[7] Cross G, and A. K. Jain (1983). Markov random field texture models. IEEE Trans. Pattern Anal. Mach. Intellegence 5. 25-39.

[8] Derin H. and W. S. Cole (1986). Segmentation of textured images using gibbs random fields. Comput. Vision Graphics image processing $35.72-98$.

[9] Derin H. and H. Elliott (1987). Modelling and segmentation of noising and textured images using Ciibbs random fields. IEEE Trans. Pattem Anal. Mach. Intelligence 9. 39-55.

[10] Galloway M. M. (1975). Texture analysis using gray level run lengths. Comput. Vision Graphics Image Processing 4. 172-179.

[11] Sun C. and W. G. Wee (1983). Neighboring gray level dependence matrix for texture classfication. Comput. Vision Graphics Image Processing 23. 341-352.

[12] Carlton $S$. G. and O. R. Nitchell (1977). Innage segmeutation using texture and gray level. IEEE Proc. Conf. on Pattern Recog. and Image Processing.

[13] Mitchell O. R., C. R. Myers, and W. Byone (1979). A max-min measure for image texture analysis. IEEE Trans. Comput. 2 $408-414$.

[14] Hsiao J. Y. and A. A. Sawchuk (1980). Unsupervised textured image segmentation using feature smoothing and probabilistic relaxation techniques. Comput. Vision Graphics Image Processing. $48.1-21$

[15] Kundu A. and J. L. Chen (1992). Texture classification using GMF bank-based subband decomposition. CVGIP: Graphical Mlodels and Image Processina 54. 369-384. 
[16] Ohanian P.P and R. C. Dubes(1992). Performance evaluation for four classes of texture features. Pattern Recognition 25.819 . 833.

[17] Tou J. T. and R. C. Gonzalez(1977). Pattern Recognition principles. Addison-Wesley Publishing Company, 377.

[18] Kande] A (1986). Fuzzy mathematical techniques with applications Addioson-Wesley Publishing Company, 274.

[19] Heermann D. P.(1992).et al, IEEE Trans. on Geoscience and Remote Sensing 30(1), 81 .

[20] Freeman A.J. and D.M. Skapura(1992). Neural netwarks, algorithms, application, and programming techniques. AddisonWesley Publishing Company.

[21] Hertz J. A. krogh, and R.G. Palmer(1991). Introduction to the theory of neural computation. Addison-Wesley Publishing Company.

[22] Rumelhart D.E., J. L. MeClelland, and PDP Group(1988). Parallel distributed processing, explorations in the microstructure of congnition, volume 1: foundations, Cambridge: MIT press.

[23] Kohonen T.(1989). Self-organization and associative menory. SV, Berlin, New York.

[24] Carpenter C. A. adn S. Grossberg(1987). A massively paralle architecture for a self-organizing neural pattern recognition machine. Comput. Vision, Graphics, Image Processing. 27, 54-115.

[25] Carpenter G. A. and S. Grossberg(1987). ART2: Selforganization of stable category recognition codes for analog input patterns, Appl. Opt. 26, 4919-4930.

[26] Carpenter (A. A and S. Grossberg(1988). The ART of adaptive pattern resognition by a self-organizing neural network, Computer, $77-88$.

[27] Carpenter G. A. adn S. Grossberg( 1990). ART3: Hierarchical search using chemical transmitters in self-organizing pattern recognition architectures, Neural Networks, 3, 129-152.

[28] Gan K.W. and K. T. Lua(1992). Chinese character classification using an adaptive resonance network. Pattern Recog. 25(8), 877882.

[29] Tiraks A, L Sukissian and S. Kollias(1990). An adaptive technique for segmentation and classification of textured inages, INNC 90 Inter. Neural Network Conf., 1, 31-34.

[30] Lu S, and A. Szeto(1991). Improving edge measurement on noisy images by hierarchical neural networks, Pattern Recog. Letters, $12,155-164$

[31] Keyvan S. and L. Rabelo (1992). Sensor signal analysis by neura] networks for surveillance in nuclear reactors, IEEE Trans. on Nuclear Science, 39(2), 292-298.

[32] Wang Z. L.(1993). IEEE Inter. Conf. an Fuzzy System, California, March

[33] Wang Z. L., G. Sylos Labini, and M. De Sario(1993).A selforganizing network of alterable competitive layer for pattern cluster, ICANN Inter. Conf. on Artificial Neural Networks. Amsterdam. The Netherlands. Sevtember.
[34] Wang Z. L.(1993).A self-organizing network of alterable competitive layer for pattern cluster, WIRN 9.96 th Italian Workshop on Netural Nets, Vietri sul Mare, Saleno, Italy, May.

[35] Wang Z. L. and M. De Sario(1993). Degign principle for artificial neural network system, Workshop on Neural Networks, Theory, Algorithms and Cognitive Modelling, Bari, Italy, June. 